\title{
BreastCare
}

\section{Breast Cancer - a Lifestyle Disease?}

\author{
Wolfgang Janni
}

Department of Obstetrics and Gynecology, University Hospital UIm, UIm, Germany

In 2017 , more than one-third (36.5\%) of US adults presented with obesity (NCHS data brief), and the estimated annual medical cost of obesity in the USA was USD 147 billion. Europe and emerging nations show the same tendency. It is long known that lifestyle factors do not only contribute substantially to the risk of developing breast cancer but also play a role in tumor progression and relapse. Both an unhealthy diet and a lack of physical activity have been reported to be associated with an increased risk of breast cancer throughout life. Furthermore, increased body weight was reported to be an independent and powerful risk factor for breast cancer. Hence, it is obvious that - if not already the case - obesity will pose a problem in the prevention as well as management of breast cancer patients.

Lifestyle interventions might therefore be able to contribute to further improving the prognosis of early breast cancer. Should lifestyle interventions prove to be consistently effective in lowering recurrence and death rates, another treatment option with an excellent benefit-risk ratio might be at hand. Patients would be able to play an active role in the disease management and could influence their individual prognosis by complying with the lifestyle recommendations. Thus, a potentially excellent benefit can be achieved due to few adverse effects of this intervention as well as its limited cost.

In the current issue of BREAST CARE with a focus on 'Lifestyle', Weigl et al. [1] present a systematic review concerning the question whether nutrition can lower the risk of recurrence in breast cancer. The authors conclude that despite the lack of conclusive data for a significant effect of nutrition on breast cancer mortality and recurrence, adherence to a healthy diet is recommended for many reasons. A prudent dietary pattern is associated with prevention of and decreased mortality from cardiovascular disease, the most common comorbidity of breast cancer survivors. Therefore, changing diet towards a healthy dietary pattern may offer multiple health benefits and improve life expectancy.

In their review article, Wirtz and Baumann [2] provide an overview on the role and evidence for rehabilitation, aftercare, physical activity, and exercise in breast cancer. They summarize that nu- merous randomized controlled trials and systematic reviews with large sample sizes showed that pre- and post-diagnosis physical activity is associated with a protective influence on recurrence and survival. Hence, the current evidence on physical activity during rehabilitation and aftercare is good but not reflected in the rehabilitation clinics.

Apart from the easily quantifiable aspects of nutrition and physical activity, Modica and Hoenig [3] stress the importance of mindfulness as part of the follow-up care of breast cancer patients. Awareness of the impact of behavior on one's health is fundamental to changing health-related behavioral patterns. In contrast, heightened awareness of possible health risks and bodily sensations might lead to increased worrisome self-observation and exaggerated health behavior. Mindfulness-Based Stress Reduction might be a valuable tool for creating a reasonable balance that allows for a prudent awareness of risks and bodily sensations as well as a good compliance with respect to follow-up care without excessive worry and social withdrawal.

In a narrative review, Lammert et al. [4] focus on opportunities for hereditary breast cancer prevention. Among BRCA mutation carriers, two independent studies showed that the receipt of a positive $B R C A 1 / 2$ genetic test result contributed to significant lifestyle changes. A prospective evaluation of multiple lifestyle behaviors, collected at various time points, with the utilization of objective methods to capture body size, physical activity, and dietary habits, is crucial to provide evidence-based, safe, and effective strategies for this high-risk group.

Within this focus on 'Lifestyle', we learn that while breast cancer definitely cannot be seen as a lifestyle disease, common environmental and behavioral patterns contribute to the risk for developing the disease as well as suffering a recurrence thereof. We should be cautious not to make feel patients guilty that the way they lived their life is mainly responsible for their disease. On the other hand, the evidence for the contributing influence of lifestyle factors is increasing and convincing. To balance our counselling based on these two perspectives should be our physician's duty, which we should be glad to accept.

\section{KARGER}

(๑) 2018 S. Karger GmbH, Freiburg

Fax +497614520714
Univ.-Prof. Dr. Wolfgang Janni 


\section{References}

1 Weigl J, Hauner H, Hauner D: Can nutrition lower the risk of recurrence in breast cancer? Breast Care 2018; 13:DOI:10.1159/000488718.

2 Wirtz P, Baumann FT: Physical activity, exercise and breast cancer - what is the evidence for rehabilitation aftercare, and survival? A review. Breast Care 2018; 13:DOI:10.1159/00088717.
3 Modica C, Hoenig K: Mindfulness in follow-up care after breast cancer: can it prevent recurrence? Breast Care 2018;13:DOI:10.1159/000488716.
4 Lammert J, Grill S, Kiechle M: Modifiable lifestyle factors: opportunities for (hereditary) breast cancer prevention - a narrative review. Breast Care 2018;13: DOI:10.1159/000488995. 